Recepción: 15 / 04 / 2017

Ciencias Médicas

Aceptación: 01 / 05 / 2017

Artículo Científico

Publicación: 15 / 05 / 2017

\title{
Reabsorción radicular durante el tratamiento de ortodoncia
}

\author{
Root resorption during orthodontic treatment
}

\section{Reabsorção radicular durante o tratamento ortodôntico}

Karen E. Tello-Falcones estefita_1592@hotmail.com

Alejandro Farfán-Chacha afarfan@uce.edu.ec

Oscar Salas-Bedon " oscar_salasb@yahoo.com

Correspondencia: estefita_1592@hotmail.com

II. Magister en Docencia Universitaria y Administración Educativa; Doctor en Odontología; Director de Investigación y Posgrado, Universidad Central del Ecuador.

III. Especialista en Ortodoncia; Doctor en Odontología, Universidad Central del Ecuador. 


\section{Resumen}

Objetivo: Identificar si existe reabsorción radicular durante el tratamiento de ortodoncia utilizando la Técnica de Roth, en los pacientes atendidos en la clínica de posgrado de la Facultad de Odontología de la Universidad Central del Ecuador periodo 2013-2015. Participantes: 40 pacientes de la Clínica de Posgrado de Ortodoncia. Material y métodos: La lectura se realizó con un calibrador digital en los incisivos centrales superiores \#11 y \#21, con el cual se midió la altura de la corona que va desde el borden incisal hasta la unión amelo cementaria; y se medió desde el borde incisal hasta el ápice.; se llevó a cabo tanto en las radiografías iniciales como finales, se dividio en cuatro grupos: pacientes con extracciones, sin extracciones, mujeres y hombres. Resultados: el valor de la prueba ANOVA (sig. $=0,000$ ) es menor que 0,05 interpretándose que existe una o algunas medidas diferentes a las otras. El valor de media más bajo es el de pacientes sin extracciones con un valor de $1,4530 \mathrm{~mm}$, se tiene un grupo central con valores estadísticamente similares pacientes mujeres y hombres con valores 2,5414 $\mathrm{mm}$ y 2,6317 mm respectivamente, al final el valor más alto es el de pacientes con extracciones con una media de 3,7110 mm. Conclusiones: Los pacientes con tratamiento de ortodoncia y con extracciones presentan mayor reabsorción radicular que los pacientes que no se han sometido a extracciones. Al relacionar los sexos hubo resultados similares, por lo que el sexo no es un factor determinante en la reabsorción radicular.

Palabras clave: Reabsorción radicular; tratamiento de ortodoncia; técnica de roth. 


\section{Abstract}

Objective: The goal of this study is to identify if there is radicular reabsorption during orthodontic treatment using the Roth Technique in patients admitted at the Post-Graduate Clinic of the School of Dentistry at Universidad Central del Ecuador, throughout the period between the years 2013 and 2015. Participants: 40 patients of the Clinic Graduate Orthodontics. Methods: The reading was performed using a digital gauge on the upper \# 11 and \# 21 central incisors, with which the crown height ranging from Borden to the junction incisal amelo cementifying was measured; and it was measured from the incisal edge to the apex; was carried out both on initial radiographs as final, was divided into four groups: patients with extractions without extractions, women and men. Results: the value of the ANOVA test (. Sig $=0,000$ ) is less than 0.05 or interpreted that there is a number of different measures to the other. The lowest average value is that of patients without extractions with a value of $1.4530 \mathrm{~mm}$, has a core group with statistically similar values female and male patients with values $2.5414 \mathrm{~mm}$ and $2.6317 \mathrm{~mm}$, respectively, at the end of the is the highest value of patients with extractions $3.7110 \mathrm{~mm}$ average. Conclusions: Patients with orthodontic treatment with extractions and root resorption have a higher than patients who have not been subjected to extractions. By linking, the sexes there were similar results, so that sex is not a determining factor in root resorption.

Key words: Radicular reabsorption; orthodontic treatment; roth technique. 


\section{Resumo}

Objetivo: o objetivo deste estudo é identificar se há reabsorção radicular durante o tratamento ortodôntico usando a Técnica Roth em pacientes admitidos na Clínica Pós-Graduação da Faculdade de Odontologia da Universidade Central do Equador, ao longo do período entre 2013 e 2015. Participantes: 40 pacientes da Clínica Pós-Graduação em Ortodontia. Métodos: A leitura foi realizada usando um indicador digital nos incisivos centrais superiores \# 11 e \# 21, com os quais foi medida a altura da coroa que varia de Borden até a junção incognoscente de amelo cementificando; E foi medido da borda incisal até o ápice; Foi realizado tanto nas radiografias iniciais como final, foi dividido em quatro grupos: pacientes com extrações sem extrações, mulheres e homens. Resultados: o valor do teste ANOVA (. Sig $=0,000)$ é inferior a 0,05 ou interpretado que há uma série de medidas diferentes para o outro. $\mathrm{O}$ valor médio mais baixo é o de pacientes sem extrações com valor de $1.4530 \mathrm{~mm}$, tem um grupo central com valores estatisticamente similares, pacientes do sexo feminino e masculino com valores de $2.5414 \mathrm{~mm}$ e $2.6317 \mathrm{~mm}$, respectivamente, no final do ano é o maior valor de pacientes Com extrações $3.7110 \mathrm{~mm}$ de média. Conclusões: pacientes com tratamento ortodôntico com extrações e reabsorção radicular têm maior que os pacientes que não foram submetidos a extrações. Ao ligar, os sexos apresentaram resultados semelhantes, de modo que o sexo não é um fator determinante na reabsorção radicular.

Palavras chave: Reabsorção radicular; tratamento ortodôntico; técnica roth. 


\section{Introducción.}

La reabsorción radicular provocada por el tratamiento de ortodoncia recientemente ha tenido considerable atención. Durante el movimiento dental la pérdida del material orgánico del ápice radicular es imprescindible, y cuando se extiende a la dentina es irreversible. Existen varios factores etiológicos como la predisposición individual, la morfología de las raíces, fuerzas aplicadas por unidad de superficie, disfunciones endocrinas, iatrogenias por partes de los profesionales y desequilibrio hormonales. (González \& Robles, 2012)

En su estudio titulado "Severa reabsorción radicular como resultado del tratamiento de ortodoncia: prevalencia y factores de riesgo", realizó un análisis mediante radiografías apicales tomadas antes y después del tratamiento. Obteniendo como resultado que los incisivos centrales superiores tiene un mayor porcentaje de reabsorción, seguidos por los incisivos laterales superiores e incisivos laterales inferiores. Teniendo en cuenta que los factores de riesgo que influyeron fueron un overjet mayor a $5 \mathrm{~mm}$, tratamientos con extracciones y un tratamiento prolongado. (Raick, Ramos, \& Vasconcellos., 2014)

Tomando en cuenta que la realización del tratamiento ortodóntico ha aumentado con los años dentro de la población; y como consecuencia de este se ha desencadenado frecuentemente una consideración clínica conocida como reabsorción radicular, se realizara este estudio con el propósito de determinar si hay reabsorción radicular en los pacientes tratados ortodónticamente. Este tema se ha elegido por la gran importancia que tiene a nivel clínico, ya que en la práctica diaria estamos expuestos a que nuestros pacientes presenten o puedan presentar dicha patología, por tal razón una vez finalizada la investigación y obtenido los resultados, se podrá determinar si el tratamiento ortodóntico utilizando la Técnica de Roth produjo reabsorción radicular en los pacientes. 


\section{Materiales y métodos.}

El presente estudio es un trabajo de investigación comparativo realizado en 40 pacientes hombres y mujeres atendidos en la Clínica de Posgrado de la Facultad de Odontología de la Universidad Central del Ecuador, en el cual se analizaron radiografías panorámicas antes y después del tratamiento de ortodoncia. También es un estudio longitudinal porque se evaluó los resultados en dos periodos de tiempo definido (2013-2015). Es un estudio observacional y descriptivo, porque no intervenimos en el fenómeno directamente, solo observamos y relatamos. El análisis de las radiografías se realizó con tres examinadores en base al criterio del examinador más dos especializados en Implantología, de los cuales estuvieron instruidos como se realizaría la valoración, primero se realizó una prueba y si los tres coincidían, se procedía a realizar el análisis definitivo. Se utilizó como materiales: calibrador digital y radiovisografo.

En este estudio se lograron estandarizar tanto la técnica radiográfica como la medición observada, lo cual permitió evitar el sesgo de información por mala clasificación.

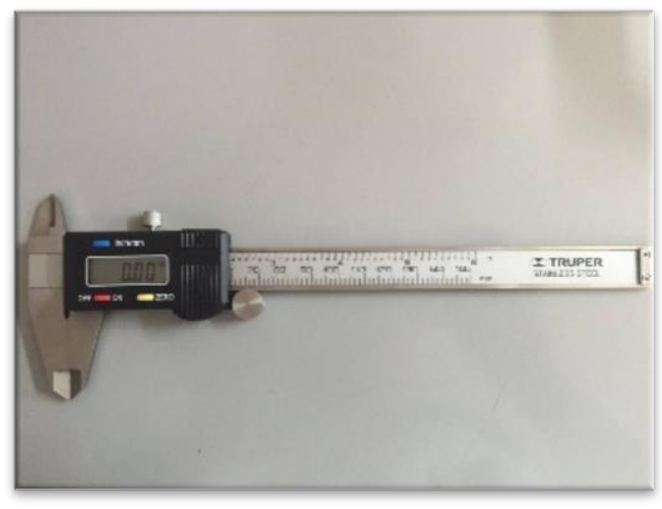

Figura No.1 Calibrador Digital 


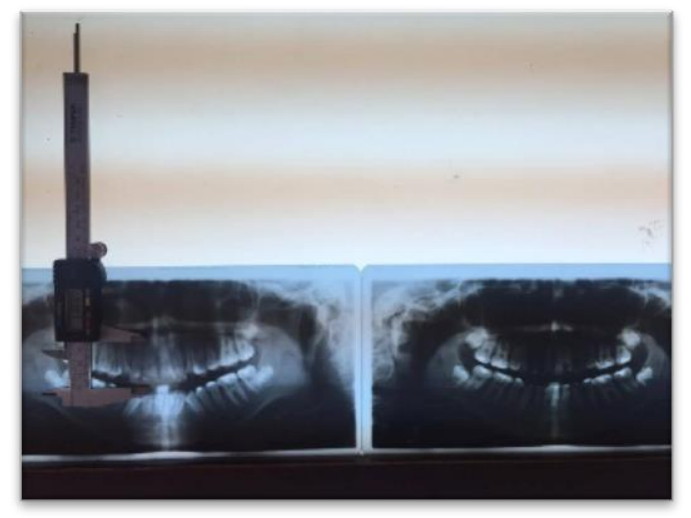

Figura No.2 Negatoscopio y Rx

Se registraron los datos de los pacientes: apellidos, nombre, $\mathrm{N}^{\mathrm{o}}$ de historia, fecha de nacimiento y fecha de obtención de la radiografía, edad y sexo. El examen radiográfico se realizó por cuadrantes y siempre en el mismo orden: primero en el primer cuadrante y luego en el segundo.

La lectura se realizó con un calibrador digital en los incisivos centrales superiores \#11 y \#21, con el cual se midió la altura de la corona que va desde el borden incisal hasta la unión amelo cementaria; y se midió desde el borde incisal hasta el ápice. Esta lectura se llevó a cabo tanto en las radiografías iniciales como finales. Para obtener los resultados se aplicó las siguientes fórmulas matemáticas:

- $\quad$ Longitud del diente (TL)

- $\quad$ Longitud de la corona (CL)

\section{1) $\mathrm{TLT}_{2}$ esperado= $\mathrm{CLT}_{2}$. $\mathrm{TLT}_{1} / \mathrm{CLT}_{1}$}

Para determinar si existe o no reabsorción se aplicó una segunda fórmula utilizando los resultados de la primera fórmula tanto de las radiografías iniciales como finales, y esta es: 


\section{2) EARRT $_{2}=$ TLT $_{2}$ esperado - TLT $_{2}$}

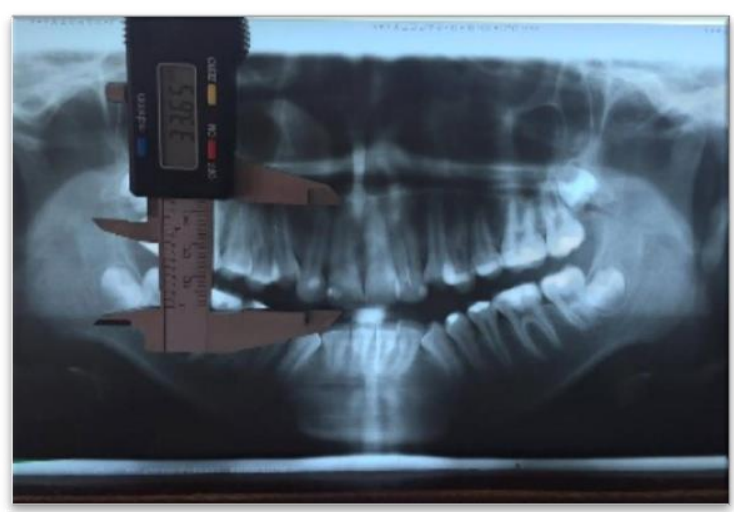

Figura No.3 Medición de la longitud del diente \#11

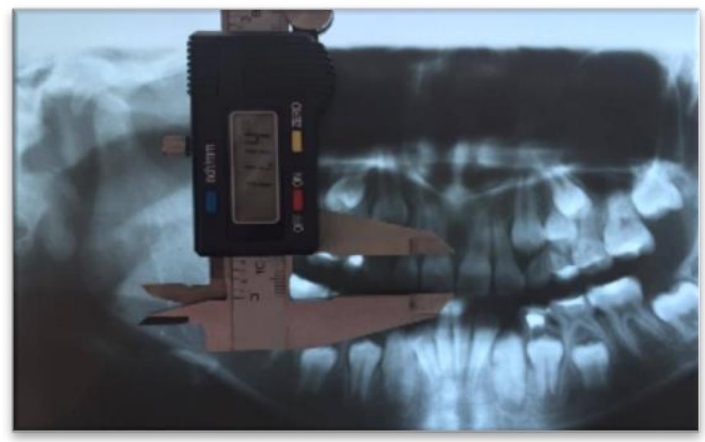

Figura No.4 Medición de la longitud de la corona del diente \#11

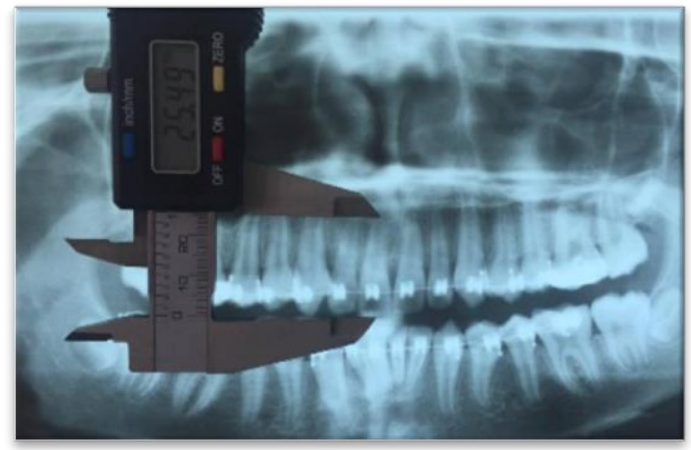

Figura No.5 Medición de la longitud del diente \#11 después de los dos años de tratamiento 


\section{Resultados}

Tabla No. 21 Resultados de la media de los tres examinadores de pacientes sin extracciones.

\begin{tabular}{|l|c|c|c|c|}
\hline Examinador & $\mathbf{1}$ & $\mathbf{2}$ & $\mathbf{3}$ & Media (M) \\
\hline Diente \#11 & 3,52 & 3.44 & 3.49 & $\mathbf{3 . 4 8}$ \\
\hline Diente \#21 & 3.71 & 3.66 & 3.75 & $\mathbf{3 . 7 0}$ \\
\hline
\end{tabular}

Tabla No.22 Resultados de la media de los tres examinadores de pacientes con extracciones.

\begin{tabular}{|l|c|c|c|c|}
\hline Examinador & $\mathbf{1}$ & $\mathbf{2}$ & $\mathbf{3}$ & Media (M) \\
\hline Diente \#11 & 1.13 & 1.27 & 1.16 & $\mathbf{1 . 1 8}$ \\
\hline Diente \#21 & 1.45 & 1.67 & 1.52 & $\mathbf{1 . 5 4}$ \\
\hline
\end{tabular}

Tabla No.23 Resultados de la media de los tres examinadores de pacientes mujeres.

\begin{tabular}{|l|c|c|c|c|}
\hline Examinador & $\mathbf{1}$ & $\mathbf{2}$ & $\mathbf{3}$ & Media (M) \\
\hline Diente \#11 & 2.07 & 2.68 & 2.16 & $\mathbf{2 . 3 0}$ \\
\hline Diente \#21 & 2.54 & 2.74 & 2.71 & $\mathbf{2 . 6 6}$ \\
\hline
\end{tabular}

Tabla No.24 Resultados de la media de los tres examinadores de pacientes hombres.

\begin{tabular}{|c|c|c|c|c|}
\hline Examinador & $\mathbf{1}$ & $\mathbf{2}$ & $\mathbf{3}$ & Media (M) \\
\hline Diente \#11 & 2.52 & 2.68 & 2.60 & $\mathbf{2 . 6 0}$ \\
\hline Diente \#21 & 2.63 & 2.73 & 2.74 & $\mathbf{2 . 7 0}$ \\
\hline
\end{tabular}

\section{Discusión}

La presente investigación tuvo como finalidad demostrar que existe reabsorción radicular durante el tratamiento orotodóntico en los pacientes de la Clínica de Posgrado de la Facultad de Odontología de la Universidad Central del Ecuador periodo 2013-2015. 
(González \& Robles, 2012) Realizaron un estudio de cohorte con seguimiento a un año, evaluando la reabsorción radicular inflamatoria con cuatro técnicas de ortodoncia. Las mediciones se realizaron en 176 dientes, usando tres radiografías periapicales por participante cada tres meses. Los datos fueron analizados a través de proporciones de incidencia. La incidencia de Reabsorción Radicular fue del $68,2 \%$, presentándose a los nueve y doce meses. Se observaron cambios radiculares con grado 1 en $8,5 \%$ de los dientes y grado 2 con $2,8 \%$, siendo laterales los de mayor proporción $(6,3 \%)$.

(Tobón, Aristizabal, \& Álverez, 2014) Realizaron un estudio donde se evaluaron 42 dientes, incisivos centrales superiores de 21 pacientes (12 mujeres, 9 hombres). Los cambios en la longitud radicular y amplitud del conducto fueron determinadas por medio de mediciones radiográficas obtenidas antes de iniciar el tratamiento (T1) y posteriormente a los 6 (T2), 12 (T3) y 18 meses (T4) utilizando un posicionador de técnica paralela RINN® XCP® (DENTSPLY) y el Sistema de radiovisiografía CDR de SCHICK ®. Obteniendo como resultado que todos los incisivos evaluados mostraron disminución de la longitud y de la amplitud del conducto radicular durante los 18 meses de tratamiento.

Los resultados obtenidos en nuestra investigación permiten demostrar que la hipótesis alternativa es aceptada, porque el valor de la prueba ANOVA (sig. $=0,000$ ) es menor que 0,05 interpretándose que existe una o algunas medidas diferentes a las otras, presentándose en el grupo de pacientes con extracciones mayor reabsorción radicular. 


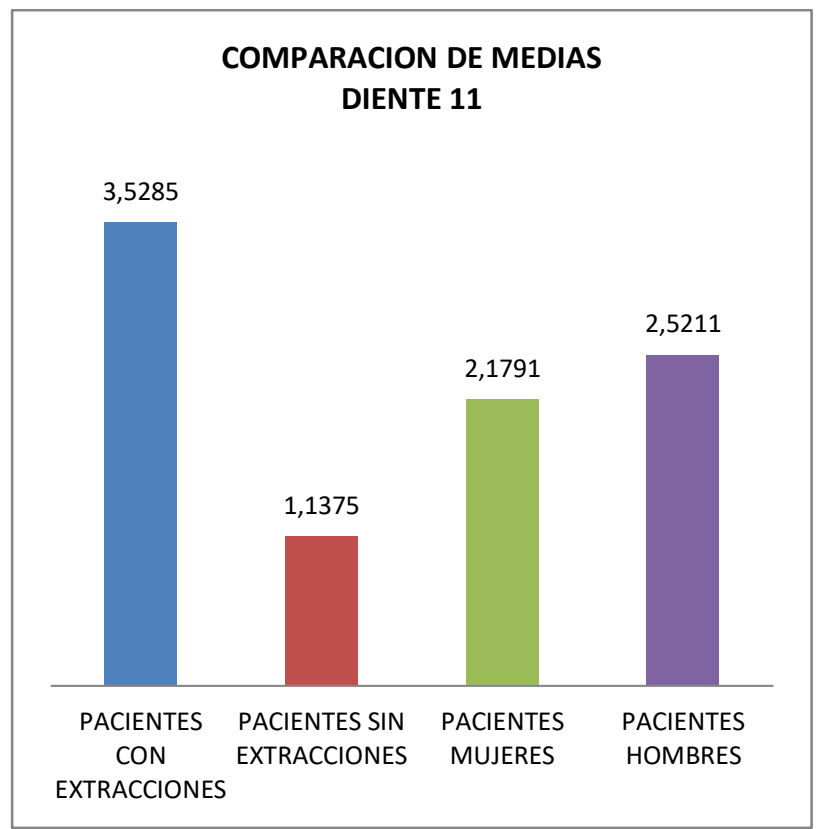

Gráfico No. 1 Gráfica comparativa de las variables del diente \#11

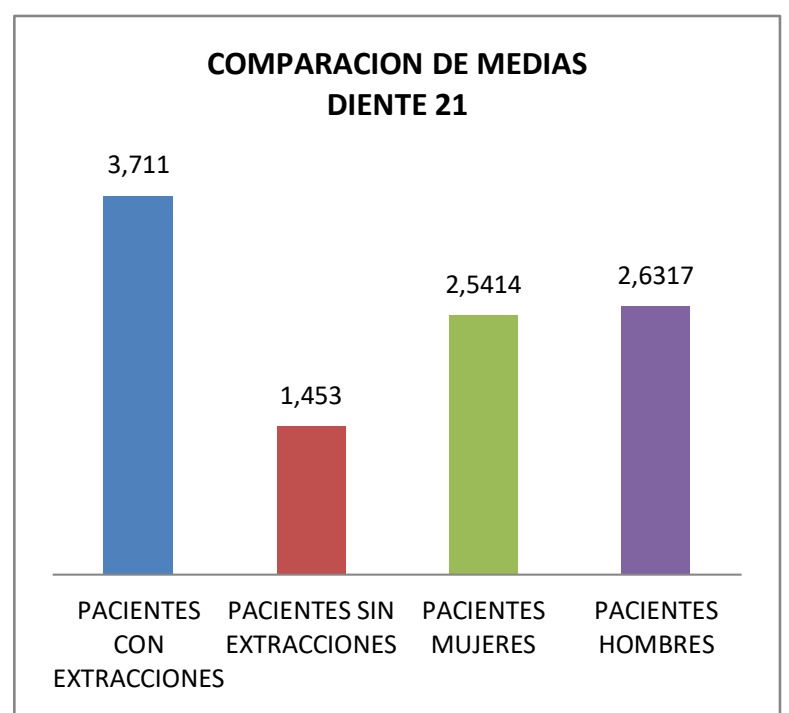

Grafico No. 2 Grafica comparativa de las variables del diente \#21

En la comparación de medias tanto del diente \#11 y \#21, el valor de la media más bajo es el de pacientes sin extracciones con un valor de 1,1375 mm (12.8\%) en el diente \#11 cuya desviación estándar es de 0,70415 mm (5,2\%) y 1,4530 (13\%) mm en el diente \#21 cuya desviación estándar es 
de $0,61129 \mathrm{~mm}(4,6 \%)$, estos valores se presentan estadísticamente similares por lo que no existe una diferencia significativa.

En la comparación de la media del grupo de pacientes mujeres y hombres con valores 2,1791 (17.6\%) mm y 2,5211 (18\%) mm para el diente \#11 y con valores 2,5414 (18,2\%) mm y 2,6317 $(18,6 \%)$ mm para el diente \#21, se presentan ambos valores estadísticamente similares por lo cual no existe una diferencia significativa

En comparación de la media con el grupo de pacientes con extracciones, presentan una media de 3,5285 (19,7\%) mm para el diente \#11 cuya desviación estándar es de 0.84260 (14\%) mm y de 3,7110 (19,9\%) mm para el diente \#21 cuya desviación estándar es de 1,07976 mm (12\%), presentándose estos valores más altos por lo que estadísticamente serán valores significativos que indican que existe una mayor reabsorción radicular.

Al comparar los cuatro grupos, el grupo de pacientes con extracciones del diente \#11, presenta una media de 3,5285 mm (19,7\%) siendo es el valor más alto de los cuatro, así como en el diente \#21 que presenta una media 3,7110 mm (19,9\%), por lo tanto se observa que de los cuatro grupos; el grupo de pacientes con extracciones tanto en hombres como en mujeres existe mayor reabsorción radicular.

\section{Conclusiones}

Con el presente estudio pude concluir:

Existe reabsorción radicular durante el tratamiento de ortodoncia, utilizando la Técnica de Roth. 
Los pacientes con tratamiento de ortodoncia y con extracciones presentan mayor reabsorción radicular que los pacientes que no se han sometido a extracciones.

Al relacionar los sexos hubo resultados similares, por lo que el sexo no es un factor determinante en la reabsorción radicular.

\section{Recomendaciones.}

Realizar un diagnóstico radiográfico más exhaustivo para controlar la cantidad de reabsorción radicular antes de empezar el tratamiento de ortodoncia.

Realizar una buena historia clínica para determinar que los diferentes factores mencionados anteriormente conlleven a una mayor reabsorción radicular.

Evitar iatrogenias durante el tratamiento de ortodoncia al aplicar determinadas fuerzas, ya que es una de las principales causas para que se produzca reabsorción radicular.

Realizar investigaciones con mayor complejidad de este tema para seguir determinando las diferentes causas durante el tratamiento de ortodoncia y posibles tratamientos que eviten que se produzca esta reabsorción.

\section{Bibliografía.}

1. Acar, A., \& Canyurek, U. (1999). Continuous vs Discontinuous force application and root resrption. Angle Orthod, 159-163.

2. Benavides, S., \& Rivera, J. (2013). Reabsorción radicular en tratamiento de Ortodoncia . Odontología Vital , 48-49 .

3. Brezniak, N., \& Wassrstein, A. (2002). Orthodontically induced inflammatory root resorption. Part I: The basic science aspects. Angle Orthod, 175-179.

4. BRUSOLA, C. (2001). Ortodoncia Clínica y Terapeútica. Barcelona: Masson, S.A. 
5. Brusola, J. C., \& Fuentes, A. S. (2001). Ortdoncia Clínica y Terapeútica . Barcelona, España : Editorial Masson, S.A.

6. Cabrini, P., Ramos, A. L., Vessoni, L., \& Micheletti, K. (2013). Analysis of correlation between inicial alveolar bone density and apical root resorption after 12 months of orthodontic treatment without extraction . Dental Press J Orthod., 97-102.

7. Capelozza, L., \& DaSilva, O. (1998). Reabsorcao radicular na clinica orthodontica: Atitudes para uma conduta preventiva . Revista Deantal de Ortodontia e Ortopedia maxilar, 104-125.

8. Caviedes, J., \& Lorenzana, T. (2002). Reabsorcion radicular externa causada por ortodoncia . Revista Universitas Odontológica, 2-6.

9. DaSilva, O., \& Capelozza, L. (1993). Estimativa da reabsorcao radicular em 50 casos ortodonticos bem finalizados . Ortodontia, volumen 26, 24-36.

10. Echave-Krutwing, M. (2002). El tratamiento ortodóntico y la reabsorcion radicular. Revisión bibliográfica . Esp Ortod , 49-55.

11. Eric, J., \& Chang, L. (2010). Apical root resorption in orthodontic patients with in-masse maxillary anterior retraction and intrusion with miniscrews. Am J of Orthodon and Dentofacial Orthop, 207212.

12. García, J., \& Varela, M. (2007). Relaciones interdisciplinares Ortodoncia-Endodoncia. Cient Dent, 185-198. 